\title{
Canadian Urological Association/Pediatric Urologists of Canada guidelines on the investigation and management of antenatally detected hydronephrosis
}

John-Paul Capolicchio, MD ${ }^{1}$; Luis H. Braga, MD²; Konrad M. Szymanski, MD, MPH ${ }^{3}$

${ }^{1}$ Montreal Children’s Hospital, McGill University Health Centre, Montreal, QC, Canada; ${ }^{2}$ McMaster Children's Hospital, McMaster University, Hamilton, ON, Canada; ${ }^{3}$ Riley Hospital for Children at Indiana University Health, Indianapolis, IN, United States

Cite as: Can Urol Assoc J 2017 Dec. 22; Epub ahead of print. http://dx.doi.org/10.5489/cuaj.5094

Published online December 22, 2017

***

\section{Introduction}

Prior to the advent of maternal ultrasonography in the 1980s, children with significant congenital hydronephrosis requiring surgery presented symptomatically with abdominal pain, urinary infection, hypertension, hematuria, or failure to thrive. Antenatal hydronephrosis (ANH) became one of the most commonly detected ultrasound findings, affecting $1-5 \%$ of pregnancies. ${ }^{1}$ The majority of ANH in the third trimester is discovered due to ultrasound for maternal indications. The benefits of this early detection of urinary tract dilation include a reduction in the renal damage due to obstruction and infection. ${ }^{2,3}$ On the other hand, many of these antenatally detected cases spontaneously resolve with observation and consequently can be submitted to unnecessary investigations and continued medical followup. ${ }^{4}$ The challenge to this day remains to predict which of these prenatally detected infants will require corrective surgery, prior to the development of symptoms or potentially irreversible renal damage, thus permitting a more tailored screening.

\section{Methodology}

This article presents an update to the 2009 guidelines(5), based on review of the current literature. The available evidence is summarized and recommendations provided based on the modified Oxford Center for Evidence-Based Medicine grading system for guideline recommendations, as employed by the International Consultation on Urologic Disease (6). 


\section{Characterizing the patient population}

The literature on ANH suffers from a lack of good quality prospective studies, which precludes any recommendations with a high level of evidence. Published prospective studies are hindered by the limitation that to this date no single gold standard diagnostic test for urinary obstruction exists. In order to appreciate this dilemma, it is imperative to understand the difference between hydronephrosis and urinary tract obstruction. Hydronephrosis simply refers to dilation of the renal collecting system. Congenital renal obstruction has been defined as “impaired urinary drainage which, if uncorrected, will limit the ultimate functional potential of the developing kidney” (7). To date, no single imaging study exists which can prove the presence of renal obstruction, contributing to the controversy surrounding management.

The differential diagnosis of ANH, in order of likelihood, includes transient primary hydronephrosis, uretero-pelvic junction obstruction (UPJO), vesicoureteric reflux (VUR), uretero-vesical junction obstruction (UVJO) or primary non-obstructive megaureter, ureterocele, ectopic ureter and causes of megacystis. Megacystis, or dilated urinary bladder, includes causes of bladder outlet obstruction due to posterior urethral valves (PUV) and less commonly Prune Belly syndrome, Megacystis-Megaureter syndrome, megacystis-microcolon intestinal hypoperistalsis syndrome (MMIHS), anterior urethral valves, megalourethra, urethral atresia and cloacal anomalies. Some of these entities have gender specific and hereditary predispositions, which have potential diagnostic implications not discussed herein.

Various grading systems for the severity of ANH exist, which are paramount in decision making. The simplest grading is the antero-posterior renal pelvic diameter (APD), which is an objective measure of the degree of pyelectasis or dilation of the renal pelvis in the transverse plane. Descriptors such as mild, moderate or severe should not be used in isolation as they are subjective and undefined. Since 1993 the standard amongst pediatric urologists in North America has been the classification from the Society for Fetal Urology (SFU), Table 1(8). The SFU grading classification has been validated with good intra-rater reliability and modest inter-rater reliability, with grade 3 being the least reliable(9). The SFU has recently proposed the UTD classification, which combines elements of both APD and SFU. Initial validation has been possibly more reliable than the SFU system $(10,11)$ with others showing the same issues with inter-rater reliability (12). The UTD classification for HN was released to address potential shortcomings of the current SFU grading system; more specifically to deal with inconsistencies between prenatally detected $\mathrm{HN}$ and postnatal management strategies within and across specialties. The UTD classification uses a 3-point system based on six different ultrasound observations [renal pelvis anterior-posterior diameter (APD), calyceal dilation, parenchymal thickness/appearance, ureteral dilation, and bladder abnormalities] to stratify patients into 3 risk categories based on the most concerning of 6 ultrasound variables (UTD P1, P2, and P3). UTD P1 (low risk) with 10-15mm renal pelvis APD and central calyceal dilation to UTD P3 with renal pelvis APD $>15 \mathrm{~mm}$, peripheral calyceal dilation, parenchymal thinning, ureter dilation, and or bladder abnormality (high risk)(13). Nevertheless, the added complexity of the UTD 
classification imposes a need for greater validation prior to supplanting the simplicity of the SFU classification.

\section{Definition of the dilated urinary tract}

The diagnosis and management of the fetus or child with a dilated urinary tract requires an understanding of what are acceptable degrees of dilation, Table 2(1). The definitions of pyelectasis, hydroureter and megacystis will determine the intensity of investigations and frequency of follow-up, both antenatally and postnatally. It is generally accepted that pyelectasis in the third trimester is defined as APD greater than 4-5 mm (1). Nevertheless, the ideal cut-off for routine postnatal screening remains controversial, since high grades of ANH can resolve postnatally and conversely low grades can deteriorate. Notwithstanding, most centres use a cutoff of $7 \mathrm{~mm}$ in the third trimester for indicating a postnatal evaluation. Postnatal data on MRI, not ultrasound, suggest that the normal APD in children is $3 \mathrm{~mm}$ at 1 year of age, $6 \mathrm{~mm}$ at 18 years with the 99th percentile for children $<5$ years of age being $<10 \mathrm{~mm}(1)$.

Aside from APD, the severity of hydroureteronephrosis (HUN) has also been classified by the SFU based on the transverse measure of the distal ureter; Grade 1 is $<7 \mathrm{~mm}$, Grade 2 is 7 to $10 \mathrm{~mm}$ and Grade 3 is $>10 \mathrm{~mm}$ (8). This classification is mostly descriptive and has not been submitted to much scrutiny. The dilated fetal bladder or megacystis has been defined based on the formula for fetal bladder sagittal length (FBSL) in mm = gestational age in weeks plus 2 (15)

It is important to remember that a dilated urinary tract does not automatically infer obstruction of the urinary tract. Whereas hydronephrosis equates to a dilated renal collecting system, obstruction cannot be proven on the basis of any single imaging study, hence the necessity for a period of observation to demonstrate deterioration over time.

\section{Antenatal vs. postnatal followup}

The frequency of ante-partum follow-up of a pregnant mother is left to the discretion of the obstetrician. While antenatal hydronephrosis (AHN) is more common in fetuses with serious chromosomal anomalies, most sources do not recommend routine karyotyping for all cases of isolated AHN. However, this may be considered in the presence of multiple system anomalies (1). Second trimester AHN is often followed up so that progression of severity can be detected and appropriate postnatal follow-up planned. Cases with severe bilateral AHN and/or oligohydramnios raise concern over potential renal failure and are often referred to the pediatric urologist for antenatal counselling. Other concerning sonographic findings include renal cortical hyper-echogenicity, renal cortical cysts and a dilated bladder. The evaluation and selection of cases for in utero intervention is beyond the scope of this guideline, but treating physicians should be aware that many tertiary care centers offer interventions, such as vesico-amniotic shunting in selected cases of bladder outlet obstruction in the setting of multidisciplinary teams. The impact of prenatal diagnosis of $\mathrm{HN}$ is also a subject of lengthy discussion; one is referred to the excellent reviews by Thomas $(3,4)$. 
Postnatal resolution has been noted in $25-50 \%$ of AHN cases $(1,16)$. Of those persisting postnatally the majority will be low grade (Table 3). In view of this, most centres will refer cases for postnatal evaluation if the third trimester APD is greater than $7 \mathrm{~mm}$, despite the fact that greater than $4 \mathrm{~mm}$ is considered abnormal by definition. Lee et al in a meta-analysis demonstrated that antenatal APD greater than $15 \mathrm{~mm}$ in the third trimester predicted an $88 \%$ chance of postnatal pathology(14). An association between higher rates of postnatal pathology and severity of HN holds true for most HN diagnoses, with the exception of VUR. VUR rates among patients with mild, moderate, and severe prenatal HN are not significantly different(14). Similarly, Dias et al have shown that if prenatal APD is $>18 \mathrm{~mm}$ in the $3^{\text {rd }}$ trimester and $>16 \mathrm{~mm}$ postnatally, the sensitivity and specificity of these cut-off values to identify infants who would eventually require pyeloplasty for UPJO were $100 \%$ and $86 \%$, respectively (17).

\section{What are the postnatal investigations?}

\section{Clinical examination}

Thorough physical examination should specifically include verifying the presence of a palpable kidney or bladder, abdominal wall abnormality, signs of spina bifida occulta, a normal introitus in females and in males the presence of gonads and a normal urethra. A baseline urinalysis can be useful in the infant follow-up period and when the child is non-verbal and unable to express symptoms of a urinary tract infection (UTI), though the need for bag specimens introduces a high risk of contamination. Serum creatinine is indicated in cases of severe bilateral HN or abnormal renal echogenicity, similarly in a solitary kidney. Serum creatinine should be obtained after 2 days to avoid confusion with maternal creatinine.

\section{Renal -bladder ultrasound (RBUS)}

All children with AHN should have a complete abdomino-pelvic ultrasound with particular attention to both the kidneys and bladder. One of the most common oversights is to focus merely on the kidneys, likely due to the fact that many radiology requisition forms separate the abdomen and pelvic ultrasounds. The RBUS should include assessment of cranio-caudal length of the kidneys, degree of echogenicity and cortico-medullary differentiation, SFU grade of hydronephrosis, maximal APD on transverse axial view of the renal pelvis, diameter of both proximal and distal ureter if dilated, the degree of bladder filling, the detrusor thickness or presence of bladder trabeculation, diverticula, ureterocele and posterior urethral dilation in males.

A full bladder should prompt a period of observation with re-imaging post void to assess for the capability to empty the bladder and to assess whether the HN improves post void. The state of bladder filling should especially be noted on serial ultrasounds and compared to the previous study when worsening $\mathrm{HN}$ is detected (19). Similarly, comparisons of renal length or APD between serial studies should be consistent with the patient positioning, as the prone views 
can differ from the supine or decubitus views (20). Fasting for a RBUS is both unnecessary and unpleasant.

Timing of the first postnatal ultrasound has been studied in a limited fashion, yet the practice standard has become to avoid doing an US in the first 2 days of life due to a concern of understaging secondary to neonatal oliguria $(1,21)$. Others have studied this issue and have not confirmed the findings(22). Certainly, in cases such as PUV where immediate postnatal management is required, there is no reason to delay the US. The acceptable delay in the timing of the first postnatal ultrasound is controversial with the SFU suggesting anywhere from 1 to 4 weeks. The timing of this study depends to a certain degree on the treating physician's attitudes to detecting asymptomatic VUR. In the absence of a desire to detect such VUR, it is intuitive that antenatal high-grade HN (HGHN, SFU grades 3-4) should be imaged soon so as to establish a baseline for serial comparison whereas low-grade HN (LGHN, SFU grades 1-2) can be imaged at a greater time interval. On the other hand, families are greatly reassured by a timelier investigation. In addition, the postnatal ultrasound may reveal subtle findings such as poor cortico-medullary differentiation, a ureterocele or detrusor hypertrophy which can easily be missed when imaging a moving fetus.

\section{Voiding cysto-urethrography}

Technical considerations are important and often overlooked in centres not accustomed to the evaluation of children (23). The study should include a scout view for assessment of spine anomalies and the presence of significant constipation or urinary stones. A balloon catheter should not be used as the balloon can obscure the filling defect characteristic of a ureterocele. The amount of urine removed should be recorded and the urine sent for analysis and culture as indicated. The bladder should be gravity filled until the first void occurs, with recording of the obtained bladder capacity. Voiding views of the urethra with postvoid views of the bladder are needed. Delayed imaging after the postvoid image may be required if there is VUR into a dilated renal pelvis or ureter so as to assess for concomitant UPJO and UVJO. A cyclical study with at least 2 fill and void cycles will increase the detection of VUR (24). Nuclear cystography is more sensitive for VUR with less radiation exposure and is generally recommended for surveillance studies or, where indicated, sibling screening.

The purpose of a VCUG is to assess for the precise cause of AHN, and is especially helpful in excluding entities such as VUR, ureteroceles and urethral anomalies such as PUV. Historical practice patterns at the onset of the era of maternal ultrasonography in the 1980s were to evaluate all infants with AHN both VCUG and nuclear renography, due to concerns over obstructive nephropathy and UTI. With experience, the yield of such studies and the natural history were better understood (14). On average, 16\% of infants with AHN are found to have VUR, with $25 \%$ of such cases occurring in the non-dilated contralateral renal unit (25). In this meta-analysis, the prevalence of VUR in a non-dilated kidney was $4 \%$, suggesting this may be the normal prevalence of VUR in the human infant. Szymanski et al compared a group of 
children with AHN screened with VCUG to a group managed with observation and demonstrated that the incidence of UTI was 1\% in LGHN (SFU Grades 1and 2) and occurred exclusively in the group who underwent VCUG (26). In this cohort, the incidence of UTI was 3fold higher in the HGHN group (SFU Grades 3 and 4) than in the LGHN group, suggesting that the grade of HN was a more important risk factor for UTI than VUR. Of note, children with bladder anomalies or renal anomalies apart from isolated HN were excluded from the study.

Certainly, any infant with suspected bladder outlet obstruction (e.g. PUV) should have an urgent VCUG. Bladder outlet obstruction would be suspected with findings of megacystis, thick or trabeculated detrusor, bilateral HGHN or dilated posterior urethra. This suspicion is amplified with findings of increased renal cortical echogenicity, renal cortical cysts or a history of oligohydramnios.

It is important to note that the clinical utility of a VCUG in HGHN is not due to concern over UTI, rather it helps to distinguish an obstructive cause of AHN from one due to VUR, thus helping to tailor the frequency and type of serial imaging studies. The American Urological Association (AUA) guidelines panel on VUR similarly recommends that VCUG be performed in infants with HGHN, hydroureter or bladder anomalies (25).

\section{Diuretic renography}

The current imaging test of choice for the assessment of the function of a hydronephrotic kidney is the MAG3 diuretic renogram, as it permits assessment of both the differential renal function (DRF) and the drainage time. Technical considerations can alter the test results, hence a standardized protocol is important in permitting comparisons of serial studies. The "welltempered renogram" was first described in 1992 by the SFU and the Society for Nuclear Medicine (SNM) (27). The protocol includes hydration to stress the urinary collecting system and bladder catheterization to avoid artifacts due to a full bladder. The SNM revised their guidelines in 2008 (28) and to this day significant differences exist compared to the protocol from the European Association of Nuclear Medicine (29). These different protocols have been compared experimentally and can lead to different interpretations of the results (30). This highlights the importance of comparing serial studies only if they were performed with the same institutional protocol.

The diuretic renogram should include a report of the DRF, which can vary between 45$55 \%$, the cortical transit time which is normal up to 5 minutes, the half-time and the shape of the curve (31). Test results can be influenced by poor renal function (single kidney GFR < 15 $\mathrm{ml} / \mathrm{min}$ ), poor hydration, massively dilated collecting system, full bladder and a dilated distal ureter (32).

Familiarity with the history of diuretic renography helps in understanding current controversies over the timing of this test. Diuretic renogram definitions of obstruction were first introduced in patients with symptomatic UPJO or a positive Whitaker test (33). When maternal ultrasonography was introduced and antenatal hydronephrosis was discovered, the same criteria were initially applied; with time the realization occurred that too many infants were having 
unnecessary pyeloplasties. In the early 1990s two landmark series of the conservative management of AHN arose which contributed to 2 schools of thought. In the Ransley group, renograms were done at 4 weeks of life due to concerns over false positive studies with immature neonatal GFR. Pyeloplasty was indicated on the basis of a single study with initial poor split function (<40\% DRF) (34). In the Koff study, renograms were performed at the time of diagnosis, regardless of age and all patients were given a chance at observation (35). The purpose of the first renogram was to serve as a baseline for serial comparison, the thinking being that drainage times would improve with renal maturation. The follow-up interval was tailored to the severity of the initially DRF in cases with low initial split function. It is interesting to note that the Koff group suffered no irreversible loss of function with observation whereas the Ransley group had a 9\% renal deterioration rate. In conclusion, renograms can be performed at any age, as long as they are used as a baseline study for serial comparison. One should also consider a DMSA study to establish early split function in cases of neonates or premature infants, since the DRF in this study is not influenced by an immature GFR (36).

\section{Ancillary tests}

Additional studies are sometimes necessary to further elucidate the precise cause of ANH, which because of their invasive nature are reserved for only select cases. Percutaneous antegrade pyelography or endoscopic retrograde pyelography can be useful when multiple levels of obstruction are suspected, such as combined UPJO and UVJO. Cystoscopy can help for evaluation of ectopic ureters and ureteroceles, similarly magnetic resonance urography (MRU). MRU can be especially helpful with the abnormal anatomy found in duplication anomalies, renal ectopy and renal fusion anomalies. This type of abnormal anatomy is often found in children with the VACTERL association or cloacal anomalies. Non-invasive studies such urinary biomarkers such as transforming growth factor beta are still under investigation, but hold promise in predicting those cases who would deteriorate and require closer follow-up (37). Similarly, the field of functional renography holds promise both with nuclear medicine (38) and positron emission tomography (39). GFR renal scan may be helpful in cases of severe bilateral HN.

\section{Continuous antibiotic prophylaxis (CAP)}

CAP has empirically been recommended for newborns with prenatal $\mathrm{HN}$ in an attempt to reduce the rate of UTI during the first two years of life. However, the AUA, the SFU and the CUA all acknowledge that use of CAP for prevention of UTI in infants with prenatal HN has been based on low levels of evidence. Not surprisingly, this lack of high-quality evidence has resulted in practice variability for CAP use. According to the 2010 SFU consensus statement on HN, CAP should be recommended only for infants with high-grade HN and those with VUR(1).

Given the uncertainty over CAP use in prenatal HN patients, a systematic review was conducted in 2013 to summarize the latest evidence regarding CAP use in children with prenatal HN. Data of nearly 4000 patients from 21 full-text articles demonstrated that pooled UTI rates 
were four times higher for high-grade HN patients when compared to those with low-grade HN. In children with low-grade HN, UTI rates were equivalent, regardless of their CAP status (2.2\% on CAP vs. 2.8\% not on CAP). On the contrary, high-grade HN patients on CAP experienced fewer UTIs than those not on CAP $(14.6 \%$ vs. 28.9\%; $\mathrm{p}<0.01)$, suggesting that CAP may be beneficial in this population. The estimated number needed to treat was seven, meaning that a clinician would offer CAP to seven patients with high-grade HN in order to prevent one UTI(40). A more recent systematic review confirmed that there seems to be value in providing CAP to infants with high-grade HN (41).

The suggestion has also been made that the presence of hydroureter or ureterocele carry a higher risk of UTI (Table 4).

The subgroup of patients with primary non-refluxing megaureters (hydroureteronephrosis) has been studied in greater detail. These infants had a much higher febrile UTI rate than those with isolated HN [19/59 (32\%) vs. 12/218 (6\%)] according to a prospective study(43). Moreover, another study demonstrated that febrile UTIs developed in $34 \%$ of megaureter patients within the first 6 months of life and that circumcision and CAP significantly decreased their infection rates(44). Other studies with retrospective design have reported similar findings(45). In addition, females and uncircumcised males with prenatal HN have also exhibited a much higher risk of UTIs(46).

The role of prophylactic antibiotics in children with prenatal HN who are awaiting completion of postnatal investigations is controversial. While it is believed that CAP may prevent UTI in children with prenatal HN, it has yet to be proven. A randomized controlled trial comparing trimethoprim to placebo in infants with SFU grades III-IV HN is currently underway in order to answer this question (47). Therefore, some authors suggest institution of CAP at birth, while others, instead, recommend a low threshold for investigation and treatment of a suspected UTI. Commonly used prophylaxes in the neonate include amoxicillin, cephalexin and trimethoprim. Trimethoprim-sulfamatholxazole and nitrofurantoin should NOT be used in the neonate because of the respective risk of kernicterus and hemolytic anemia.

\section{Followup protocols}

\section{SFU Grades 3 and 4, APD >15 mm, HGHN}

The initial postnatal RBUS should be done as soon as feasible after day 2 of life, as this represents the cohort most likely to require surgery and to experience UTI. The likelihood of grade $4 \mathrm{HN}$ undergoing a pyeloplasty is up to 75\%(35). Most centres recommend the US within 2 weeks of life. Bilateral HGHN requires more urgent consultation, including a VCUG to exclude PUV. If the postnatal US reveals persistent HGHN, these cases should be referred to a Pediatric Urologist for immediate consultation. These cases are most likely to benefit from CAP and should have both a VCUG and MAG3 renal scan. It is worth repeating that the clinical utility of a VCUG in HGHN is not due to concern over UTI, rather it helps to distinguish an obstructive cause of AHN from one due to VUR, thus helping to tailor the frequency and type of serial 
imaging studies. In the absence of any pathology requiring immediate intervention, repeat US and MAG3 should be performed within 3 months, though a repeat MAG3 is optional if the first exam is normal (Algorithm). Close follow-up should continue if improvement is not seen, to at least 18 months of age by which time most childhood UPJO becomes apparent. One should keep in mind that historical cohorts of symptomatic UPJO prior to the advent of maternal ultrasonography would undergo pyeloplasty at an average age of 6 years (2), hence a persistent grade $3 \mathrm{HN}$ requires active surveillance.

\section{SFU Grade 1 and 2, APD $<10 \mathrm{~mm}, \mathrm{LGHN}$}

The timing of the first postnatal US is open to debate and left to the discretion of the treating physician. For cases with antenatal APD between $10-15 \mathrm{~mm}$ the SFU grading is suggested to clarify which follow-up protocol should be used. Most physicians will obtain an ultrasound within the first months of life and a follow-up can be obtained 6 months later. In the absence of deterioration, follow-up US can then be performed on an annual basis. VCUG and Mag3 are not required. Szymanski et al demonstrated the risk of UTI in isolated LGHN with no renal size discrepancy and no ureteral or bladder abnormalities to be less than $1 \%$ (26), hence CAP is unnecessary. The majority of such cases improve by 2 years of age (48). Since the majority of congenital UPJO progresses to pyeloplasty by 18 months of age it seems prudent to continue the follow-up to at least such an age. Multiple authors have shown that with long-term follow-up to 10 years the risk of deterioration requiring surgical intervention to be $2 \%(49,50)$. The question then arises as to the need for follow-up beyond 2 years of age in patients who have persistent LGHN. Some authors recommend discharge and counsel families on the symptoms of UPJO. Future prospective studies will be needed to determine the most cost-effective and clinically appropriate follow-up protocol for children with prenatal HN. Akhavan et al. have looked at the resource utilization associated with the diagnostic evaluation of nonrefluxing HN infants and found that decreasing the number of US performed during follow-up for patients with SFU grades I-II HN could reduce $24 \%$ of the health care costs(51).

\section{SFU Grade 0}

Up to $50 \%$ of AHN can resolve at birth and is referred to as SFU Grade $0 \mathrm{HN}$. Such transient $\mathrm{HN}$ is also the subject of controversy with the SFU guidelines recommending a repeat US within the first month of life (1). Many centres will discharge the patient after a normal postnatal US, since the majority of late or recurrent $\mathrm{HN}$ are symptomatic and can be counselled of such. Certainly, it appears contradictory to follow-up a documented LGHN within 6 months whereas a normal US is followed-up within 1 month.

\section{Indications for surgery in obstructive HN and HUN}

The current difficulty in proving the presence of obstruction in HN and HUN imply that a period of observation is needed to document deterioration. This period of observation carries a risk of potentially irreversible loss of renal function (52). For this reason, appropriate counselling of 
families should include a discussion of the risks of observation versus immediate surgery. Strong indications for reconstructive surgery include loss of DRF of greater than $5 \%$ on serial renography or worsening $\mathrm{HN}$ with worsening drainage times on renography. In older children flank pain or vomiting are also suggestive of obstruction, especially if exacerbated by fluid intake. Hypertension and renal calculi can rarely be signs of obstruction. Relative indications for surgery include UTI, low DRF on initial renogram, palpable giant HN, concern over noncompliance with follow-up imaging protocols and family preference in cases of persistent HGHN requiring repeated renographic evaluation. In one study an alarming $42 \%$ of children with HGHN were lost to follow-up (53). The threshold for surgical intervention is lowered in cases of solitary kidney or bilateral HGHN.

\section{Summary of recommendations}

1. All significant AHN should be investigated with a postnatal RBUS. Most centres define significant AHN in the third trimester as APD $\geq 7 \mathrm{~mm}$ (Level 3 evidence; Grade $\mathbf{C}$ recommendation).

2. The role of CAP initiated at birth is controversial, but may be of greater benefit in grades 3 and $4 \mathrm{HN}$ and in cases with dilated ureter or bladder abnormality. Females and uncircumcised males with AHN may also benefit more compared to circumcised boys (Level 3 evidence; Grade $C$ recommendation).

3. VCUG is not necessary in the evaluation of isolated low grade (SFU 1 and 2) HN with normal renal parenchyma and symmetric renal size (Level 3 evidence; Grade C recommendation).

4. High grade HN (SFU III-IV) should be investigated with a VCUG, followed by diuretic renography if the HN cannot be explained by VUR (Level 4 evidence; Grade D recommendation).

5. Diuretic renography is not necessary in the evaluation of isolated low grade (SFU 1 and 2) HN with normal renal parenchyma and symmetric renal size (Level 4 evidence;

\section{Grade D recommendation).}


Table of Abbreviations:

HN - Hydronephrosis

AHN - Antenatal hydronephrosis

SFU - Society for Fetal Urology

HGHN - High grade HN, SFU Grade 3-4

LGHN - Low grade HN, SFU Grade 1-2

APD - Antero-posterior renal pelvic diameter

UPJO - Uretero-pelvic junction obstruction

UVJO - Uretero-vesical junction obstruction

VUR - Vesicoureteric reflux

PUV - Posterior urethral valve

RBUS - Renal-bladder ultrasound

VCUG - Voiding cysto-urethrogram

DRF - Differential renal function

CAP - Continuous antibiotic prophylaxis

MAG3 - Tc-99m mercaptoacetyltriglycine

DMSA- Dimercaptosuccinic acid

PUC - Pediatric Urologists of Canada

CUA - Canadian Urological Association

AUA - American Urological Association

UTI - Urinary Tract Infection 


\section{References:}

1. Nguyen HT, Herndon CD, Cooper C, Gatti J, Kirsch A, Kokorowski P, et al. The Society for Fetal Urology consensus statement on the evaluation and management of antenatal hydronephrosis. J Pediatr Urol. 2010;6(3):212-31.

2. Capolicchio G, Leonard MP, Wong C, Jednak R, Brzezinski A, Salle JL. Prenatal diagnosis of hydronephrosis: impact on renal function and its recovery after pyeloplasty. J Urol. 1999;162(3 Pt 2):1029-32.

3. Thomas DF. Prenatal diagnosis: does it alter outcome? Prenat Diagn. 2001;21(11):100411.

4. Thomas DF. Prenatal diagnosis: what do we know of long-term outcomes? J Pediatr Urol. 2010;6(3):204-11.

5. Psooy K, Pike J. Investigation and management of antenatally detected hydronephrosis. Can Urol Assoc J. 2009;3(1):69-72.

6. Abrams P, Khoury S, Grant A. Evidence--based medicine overview of the main steps for developing and grading guideline recommendations. Prog Urol. 2007;17(3):681-4.

7. Peters CA. Urinary tract obstruction in children. J Urol. 1995;154(5):1874-83; discussion 83-4.

8. $\quad$ Fernbach SK, Maizels M, Conway JJ. Ultrasound grading of hydronephrosis: introduction to the system used by the Society for Fetal Urology. Pediatr Radiol. 1993;23(6):478-80.

9. Keays MA, Guerra LA, Mihill J, Raju G, Al-Asheeri N, Geier P, et al. Reliability assessment of Society for Fetal Urology ultrasound grading system for hydronephrosis. J Urol. 2008;180(4 Suppl):1680-2; discussion2-3.

10. Hodhod A, Capolicchio JP, Jednak R, El-Sherif E, El-Doray Ael A, El-Sherbiny M. Evaluation of Urinary Tract Dilation Classification System for Grading Postnatal Hydronephrosis. J Urol. 2016;195(3):725-30.

11. Chalmers DJ, Meyers ML, Brodie KE, Palmer C, Campbell JB. Inter-rater reliability of the APD, SFU and UTD grading systems in fetal sonography and MRI. J Pediatr Urol. 2016.

12. Rickard M, Easterbrook B, Kim S, Farrokhyar F, Stein N, Arora S, et al. Six of one, half a dozen of the other: A measure of multidisciplinary inter/intra-rater reliability of the society for fetal urology and urinary tract dilation grading systems for hydronephrosis. J Pediatr Urol. 2017;13(1):80 e1- e5.

13. Nguyen HT, Benson CB, Bromley B, Campbell JB, Chow J, Coleman B, et al. Multidisciplinary consensus on the classification of prenatal and postnatal urinary tract dilation (UTD classification system). J Pediatr Urol. 2014;10(6):982-98.

14. Lee RS, Cendron M, Kinnamon DD, Nguyen HT. Antenatal hydronephrosis as a predictor of postnatal outcome: a meta-analysis. Pediatrics. 2006;118(2):586-93. 
15. Maizels M, Alpert SA, Houston JT, Sabbagha RE, Parilla BV, MacGregor SN. Fetal bladder sagittal length: a simple monitor to assess normal and enlarged fetal bladder size, and forecast clinical outcome. J Urol. 2004;172(5 Pt 1):1995-9.

16. Barbosa JA, Chow JS, Benson CB, Yorioka MA, Bull AS, Retik AB, et al. Postnatal longitudinal evaluation of children diagnosed with prenatal hydronephrosis: insights in natural history and referral pattern. Prenat Diagn. 2012;32(13):1242-9.

17. Dias CS, Silva JM, Pereira AK, Marino VS, Silva LA, Coelho AM, et al. Diagnostic accuracy of renal pelvic dilatation for detecting surgically managed ureteropelvic junction obstruction. J Urol. 2013;190(2):661-6.

18. Ahmad G, Green P. Outcome of fetal pyelectasis diagnosed antenatally. J Obstet Gynaecol. 2005;25(2):119-22.

19. Leung VY, Chu WC, Metreweli C. Hydronephrosis index: a better physiological reference in antenatal ultrasound for assessment of fetal hydronephrosis. J Pediatr. 2009;154(1):116-20.

20. Carrico CW, Zerin JM. Sonographic measurement of renal length in children: does the position of the patient matter? Pediatr Radiol. 1996;26(8):553-5.

21. Dejter SW, Jr., Gibbons MD. The fate of infant kidneys with fetal hydronephrosis but initially normal postnatal sonography. J Urol. 1989;142(2 Pt 2):661-2; discussion 7-8.

22. Docimo SG, Silver RI. Renal ultrasonography in newborns with prenatally detected hydronephrosis: why wait? J Urol. 1997;157(4):1387-9.

23. Frimberger D, Mercado-Deane MG, Section On U, Section On R. Establishing a Standard Protocol for the Voiding Cystourethrography. Pediatrics. 2016;138(5).

24. Jequier S, Jequier JC. Reliability of voiding cystourethrography to detect reflux. AJR Am J Roentgenol. 1989;153(4):807-10.

25. Skoog SJ, Peters CA, Arant BS, Jr., Copp HL, Elder JS, Hudson RG, et al. Pediatric Vesicoureteral Reflux Guidelines Panel Summary Report: Clinical Practice Guidelines for Screening Siblings of Children With Vesicoureteral Reflux and Neonates/Infants With Prenatal Hydronephrosis. J Urol. 2010;184(3):1145-51.

26. Szymanski KM, Al-Said AN, Pippi Salle JL, Capolicchio JP. Do infants with mild prenatal hydronephrosis benefit from screening for vesicoureteral reflux? J Urol. 2012;188(2):576-81.

27. Conway JJ, Maizels M. The "well tempered" diuretic renogram: a standard method to examine the asymptomatic neonate with hydronephrosis or hydroureteronephrosis. A report from combined meetings of The Society for Fetal Urology and members of The Pediatric Nuclear Medicine Council--The Society of Nuclear Medicine. J Nucl Med. 1992;33(11):2047-51. 28. Shulkin BL, Mandell GA, Cooper JA, Leonard JC, Majd M, Parisi MT, et al. Procedure guideline for diuretic renography in children 3.0. J Nucl Med Technol. 2008;36(3):162-8. 
29. Gordon I, Piepsz A, Sixt R, Auspices of Paediatric Committee of European Association of Nuclear M. Guidelines for standard and diuretic renogram in children. Eur J Nucl Med Mol Imaging. 2011;38(6):1175-88.

30. De Man KE, Troch ME, Dobbeleir AA, Duong HP, Goethals IM. Comparison of the EANM and SNM guidelines on diuretic renography in children. Nucl Med Commun. 2015;36(5):486-8.

31. Capolicchio G, Jednak R, Dinh L, Salle JL, Brzezinski A, Houle AM. Supranormal renographic differential renal function in congenital hydronephrosis: fact, not artifact. J Urol. 1999;161(4):1290-4.

32. Rao NP, Srirangam SJ, Preminger GM. Nuclear Medicine Investigations. Urological Tests in Clinical Practice. London: Springer-Verlag; 2007. p. 132-62.

33. Kass EJ, Majd M. Evaluation and management of upper urinary tract obstruction in infancy and childhood. Urol Clin North Am. 1985;12(1):133-41.

34. Ransley PG, Dhillon HK, Gordon I, Duffy PG, Dillon MJ, Barratt TM. The postnatal management of hydronephrosis diagnosed by prenatal ultrasound. J Urol. 1990;144(2 Pt 2):5847; discussion 93-4.

35. Koff SA, Campbell K. Nonoperative management of unilateral neonatal hydronephrosis. J Urol. 1992;148(2 Pt 2):525-31.

36. Sfakianakis GN, Sfakianaki E. Renal scintigraphy in infants and children. Urology. 2001;57(6):1167-77.

37. Almodhen F, Loutochin O, Capolicchio JP, Jednak R, El-Sherbiny M. The role of bladder urine transforming growth factor-beta1 concentrations in diagnosis and management of unilateral prenatal hydronephrosis. J Urol. 2009;182(1):292-8; discussion 8.

38. Homsy YL, Tripp BM, Lambert R, Campos A, Capolicchio G, Dinh L, et al. The captopril renogram: a new tool for diagnosing and predicting obstruction in childhood hydronephrosis. J Urol. 1998;160(4):1446-9.

39. Szabo Z, Xia J, Mathews WB, Brown PR. Future direction of renal positron emission tomography. Semin Nucl Med. 2006;36(1):36-50.

40. Braga LH, Mijovic H, Farrokhyar F, Pemberton J, DeMaria J, Lorenzo AJ. Antibiotic prophylaxis for urinary tract infections in antenatal hydronephrosis. Pediatrics. 2013;131(1):e251-61.

41. Easterbrook B, Capolicchio JP, Braga LH. Antibiotic prophylaxis for prevention of urinary tract infections in prenatal hydronephrosis: An updated systematic review. Can Urol Assoc J. 2017;11(1-2Suppl1):S3-S11.

42. Lee JH, Choi HS, Kim JK, Won HS, Kim KS, Moon DH, et al. Nonrefluxing neonatal hydronephrosis and the risk of urinary tract infection. J Urol. 2008;179(4):1524-8.

43. Braga LH, Farrokhyar F, D'Cruz J, Pemberton J, Lorenzo AJ. Risk factors for febrile urinary tract infection in children with prenatal hydronephrosis: a prospective study. J Urol. 2015;193(5 Suppl):1766-71. 
44. Braga LH, D'Cruz J, Rickard M, Jegatheeswaran K, Lorenzo AJ. The Fate of Primary Nonrefluxing Megaureter: A Prospective Outcome Analysis of the Rate of Urinary Tract Infections, Surgical Indications and Time to Resolution. J Urol. 2016;195(4 Pt 2):1300-5.

45. Herz D, Merguerian P, McQuiston L. Continuous antibiotic prophylaxis reduces the risk of febrile UTI in children with asymptomatic antenatal hydronephrosis with either ureteral dilation, high-grade vesicoureteral reflux, or ureterovesical junction obstruction. J Pediatr Urol. 2014;10(4):650-4.

46. Zee RS, Herbst KW, Kim C, McKenna PH, Bentley T, Cooper CS, et al. Urinary tract infections in children with prenatal hydronephrosis: A risk assessment from the Society for Fetal Urology Hydronephrosis Registry. J Pediatr Urol. 2016;12(4):261 e1-7.

47. Braga LH, Pemberton J, Heaman J, DeMaria J, Lorenzo AJ. Pilot randomized, placebo controlled trial to investigate the effect of antibiotic prophylaxis on the rate of urinary tract infection in infants with prenatal hydronephrosis. J Urol. 2014;191(5 Suppl):1501-7.

48. Zee RS, Herndon CDA, Cooper CS, Kim C, McKenna PH, Khoury A, et al. Time to resolution: A prospective evaluation from the Society for Fetal Urology hydronephrosis registry. J Pediatr Urol. 2017;13(3):316 e1- e5.

49. Matsui F, Shimada K, Matsumoto F, Takano S. Late recurrence of symptomatic hydronephrosis in patients with prenatally detected hydronephrosis and spontaneous improvement. J Urol. 2008;180(1):322-5; discussion 5.

50. Sidhu G, Beyene J, Rosenblum ND. Outcome of isolated antenatal hydronephrosis: a systematic review and meta-analysis. Pediatr Nephrol. 2006;21(2):218-24.

51. Akhavan A, Shnorhavorian M, Garrison LP, Jr., Merguerian PA. Resource utilization and costs associated with the diagnostic evaluation of nonrefluxing primary hydronephrosis in infants. J Urol. 2014;192(3):919-24.

52. Dhillon HK. Prenatally diagnosed hydronephrosis: the Great Ormond Street experience. Br J Urol. 1998;81 Suppl 2:39-44.

53. Cordero L, Nankervis CA, Oshaughnessy RW, Koff SA, Giannone PJ. Postnatal followup of antenatal hydronephrosis: a health-care challenge. J Perinatol. 2009;29(5):382-7. 


\section{Figures and Tables}

Fig. 1. Algorithm for management of antenatal hydronephrosis.

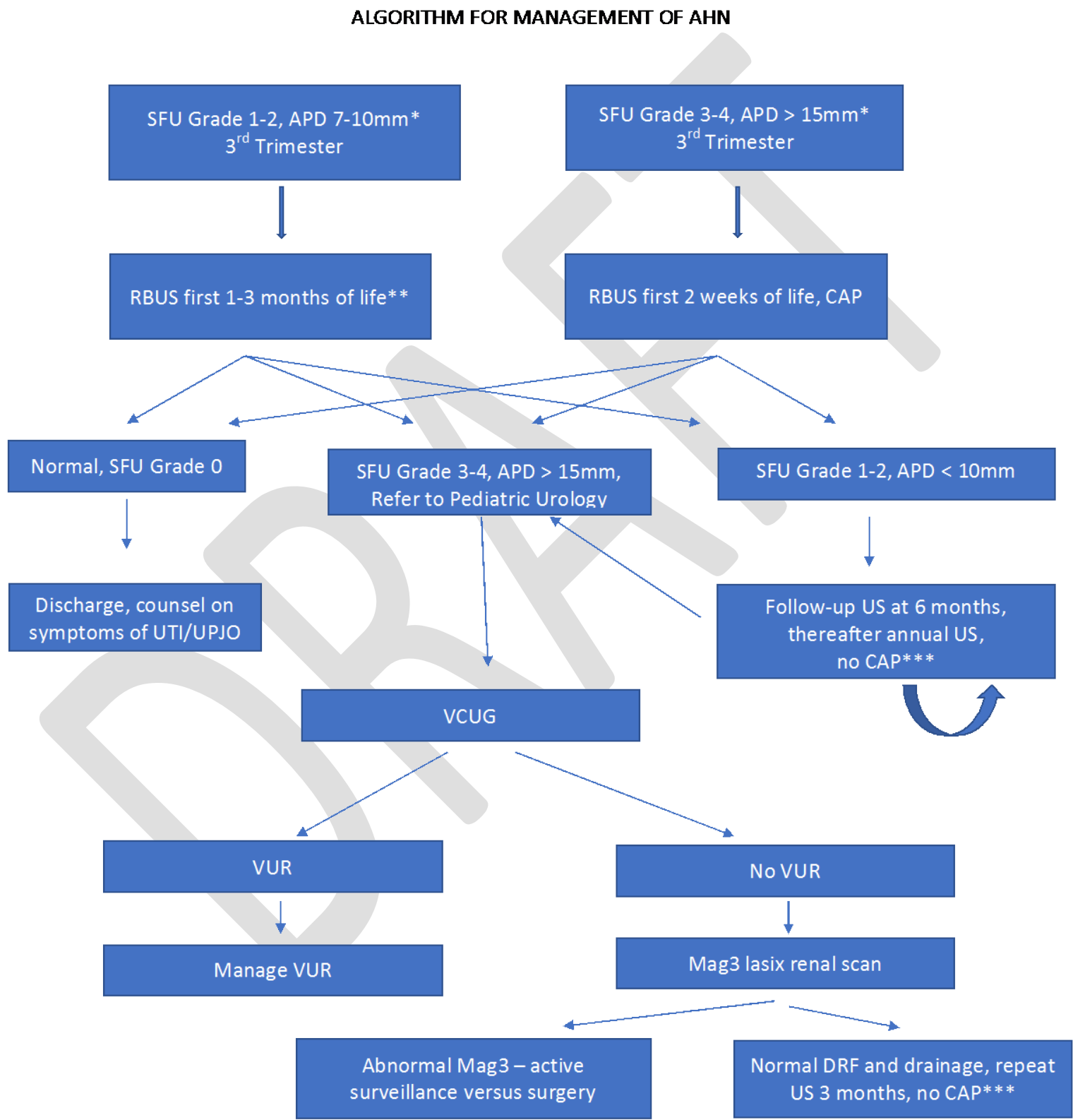

* APD between 10-15 $\mathrm{mm}$ should be managed by the SFU grade.

** Dilated ureters, abnormal bladders or abnormal renal parenchyma should be imaged sooner.

*** Some authors advocate CAP for LGHN with dilated ureters or abnormal bladders. The risk of UTI is also increased in females and un-circumcised males. 


\begin{tabular}{|l|l|}
\hline \multicolumn{2}{|l|}{ Table 1. SFU grading of hydronephrosis } \\
\hline SFU grade & Ultrasound findings \\
\hline $\mathbf{0}$ & Normal kidney (resolved antenatal hydronephrosis) \\
\hline $\mathbf{1}$ & Pyelectasis \\
\hline $\mathbf{2}$ & Pyelectasis with dilation of 1 or more major calyces (caliectasis) \\
\hline $\mathbf{3}$ & Pyelectasis with dilation of all 3 major calyces \\
\hline $\mathbf{4}$ & Pyelectasis with parenchymal thinning compared to contralateral kidney \\
\hline
\end{tabular}

SFU: Society for Fetal Urology.

\begin{tabular}{|l|c|c|}
\hline Table 2. Severity of antenatal hydronephrosis (ANH) by APD \\
\hline Degree of ANH & Second trimester & Third trimester \\
\hline Mild & 4 to $<7 \mathrm{~mm}$ & 7 to $<9 \mathrm{~mm}$ \\
\hline Moderate & 7 to $\leq 10 \mathrm{~mm}$ & 9 to $\leq 15 \mathrm{~mm}$ \\
\hline Severe & $>10 \mathrm{~mm}$ & $>15 \mathrm{~mm}$ \\
\hline
\end{tabular}

APD: antero-posterior renal pelvic diameter.

Table 3. Distribution of antenatal hydronephrosis (ANH) severity and likelihood of postnatal urinary tract pathology ${ }^{18}$

\begin{tabular}{|l|c|c|}
\hline Degree of ANH & \% of ANH & \% postnatal pathology \\
\hline Mild & $57-88$ & 12 \\
\hline Moderate & $10-30$ & 45 \\
\hline Severe & $1.5-13$ & 88 \\
\hline
\end{tabular}




\begin{tabular}{|l|c|c|c|c|}
\hline \multicolumn{5}{|c|}{ Table 4. Incidence of urinary tract infections (UTIs) in patients with hydronephrosis ${ }^{\mathbf{4 2}}$} \\
\hline & No. UTI (\%) & p & OR & 95\% CI \\
\hline Sex & & & & \\
\hline Male & $67(19)$ & 0.8 & 0.93 & $0.51-1.71$ \\
\hline Female & $16(20)$ & & & \\
\hline Obstruction & & & & \\
\hline Yes & $50(39)$ & 0.001 & 5.23 & $3.148-8.67$ \\
\hline No & $33(11)$ & & & \\
\hline Hydroureter & & & & \\
\hline Yes & $37(47)$ & 0.001 & 6.00 & $3.49-10.32$ \\
\hline No & $46(13)$ & & & \\
\hline Uretetocele & & & & \\
\hline Yes & $10(59)$ & 0.001 & 6.65 & $2.45-18.06$ \\
\hline No & $73(18)$ & & & \\
\hline HN grade & & & & \\
\hline I & $6(4)$ & & & \\
\hline II & $13(14)$ & 0.001 & 4.15 & $1.52-11.32$ \\
\hline III & $26(33)$ & 0.001 & 12.67 & $4.95-32.47$ \\
\hline IV & $38(40)$ & 0.001 & 16.93 & $6.80-42.15$ \\
\hline
\end{tabular}

